are not bounded. It follows that $f^{\prime}(t)$ does not exist, as was to be proved.

\title{
REFERENCES
}

1. S. Banach, Théorie des opérations linéaires, Warsaw, 1932.

2. E. W. Hobson, The theory of functions of a real variable, vol. 2, Cambridge, 1926.

3. S. N. Mergelyan, Uniform approximations to functions of a complex variable, Amer. Math. Soc. Translation no. 101, Providence, 1954.

University of California, Berkeley

\section{AN IDENTITY IN THE THEORY OF THE GENERALIZED POLYNOMIALS OF JACOBI}

\section{B. GERMANSKY}

1. Introduction of some new notations in the theory of the Jacobi polynomials. To facilitate the passage from the usual Jacobi polynomials $P_{n}^{(\alpha, \beta)}(x)$ to the generalized Jacobi polynomials $P_{n}^{\left(\alpha_{0}, \ldots, \alpha_{p}\right)}(x)$ considered here, we introduce some new notations in the theory of the first mentioned polynomials. It is well known ${ }^{1}$ that the zeros of these polynomials are the points $x_{1}=x_{1}^{(n)}, x_{2}=x_{2}^{(n)}, \cdots, x_{n}=x_{n}^{(n)}$, which maximize the expression

$$
T\left(x_{1}, x_{2}, \cdots, x_{n}\right)=T(x)=\prod_{k=1}^{n}\left(1-x_{k}\right)^{p}\left(1+x_{k}\right)^{q} \prod_{1 \leqq \nu<\mu \leqq n}\left|x_{\nu}-x_{\mu}\right|
$$

in the unit-interval $I:[-1,+1]$. Here $\alpha=2 p-1$ and $\beta=2 q-1$ and it is assumed $x_{1}>x_{2}>\cdots>x_{n}$. Instead of $T(x)$ we use the expression

$$
V_{m}\left(\xi_{1}, \xi_{2}, \cdots, \xi_{m} ; e_{1}, e_{2}, \cdots, e_{m}\right)=V_{m}(\xi ; e)=\prod_{1 \leqq i<k \$ m}\left(\xi_{i}-\xi_{k}\right)^{e_{i e k}},
$$

where we suppose that $m=n+2$; that the points $\xi_{1}$ and $\xi_{m}$ are fixed from the outset and are equal to $a_{0}=-1$ and $a_{1}=+1$ respectively; that $e_{1}=\rho_{0}=q, e_{m}=\rho_{1}=p, e_{2}=e_{3}=\cdots=e_{m-1}=1$; that the points $\xi_{1}, \xi_{2}, \cdots, \xi_{m}$ are counted in increasing order; $-1=\xi_{1}<\xi_{2}<\ldots$ $<\xi_{m-1}<\xi_{m}=+1$ and therefore that $\xi_{2}=x_{n}, \xi_{3}=x_{n-1}, \cdots, \xi_{m-1}=x_{1}$. It results that $V_{m}\left(\xi_{1}, \xi_{2}, \cdots, \xi_{m} ; e_{1}, e_{2}, \cdots, e_{m}\right)$ is a function of $\xi_{2}, \xi_{3}, \cdots, \xi_{m-1} ; \rho_{0}, \rho_{1}$ only, as is $T(x)$. Then the zeros of the Jacobi polynomial $P_{n}^{(\alpha, \beta)}(x)$ are the points $\xi_{2}=\xi_{2}^{(m)}=x_{n}^{(n)}, \xi_{3}=\xi_{3}^{(m)}=x_{n-1}^{(n)}, \cdots$, $\xi_{m-1}=\xi_{m-1}^{(m)}=x_{1}^{(n)}$, which maximize the absolute value of $V_{m}(\xi ; e)$ on $I$, under the mentioned conditions. We call the last function the generalized Vandermondean of the degree $m$ and of the order 1 . We write

Received by the editors January 2, 1958 and, in revised form, April 25, 1958.

${ }^{1}$ Szegö, Orthogonal polynomials, New York, 1939, p. 136, Theorem 6.7.1. 
the Jacobi polynomial $P_{n}^{(\alpha, \beta)}(x)$ also in the form $P_{n}^{\left(\alpha_{0}, \alpha_{1}\right)}(x),{ }^{2}$ and call it the Jacobi polynomial of the degree $n$ and of the order 1 . Here $\alpha_{0}=\beta$ and $\alpha_{1}=\alpha$, and we suppose $x_{1}<x_{2}<\cdots<x_{n}{ }^{3}$

For later use we introduce also the function $p_{m}^{\left(\alpha_{0}, \alpha_{1}\right)}(x)$ $=(x+1)^{\alpha_{0}} P_{n}^{\left(\alpha_{0}, \alpha_{1}\right)}(x)(x-1)^{\alpha_{1}}$, the zeros of which are $\xi_{1}^{(m)}=-1$, $\xi_{2}^{(m)}, \cdots, \xi_{m-1}^{(m)}, \xi_{m}^{(m)}=+1$. We call, for the sake of simplicity, also functions of the form $\left(x-\xi_{1}\right)^{e_{1}}\left(x-\xi_{2}\right)^{e_{2}} \cdots\left(x-\xi_{m}\right)^{e_{m}}$ (generalized) polynomials and will omit the word "generalized". The polynomials $p_{m}^{\left(\alpha_{0}, \alpha_{1}\right)}(x)$ are called by us extended Jacobi polynomials.

2. The generalized Jacobi polynomials. We consider now the generalized Vandermondean $V_{m}\left(\xi_{1}, \xi_{2}, \cdots, \xi_{m} ; e_{1}, e_{2}, \cdots, e_{m}\right)$ under the conditions that instead of the two fixed points $\xi_{1}=a_{0}=-1$ and $\xi_{m}=a_{1}=+1$, loaded, by the electrostatic interpretation of the whole problem, ${ }^{4}$ with the charges (weights) $e_{1}=\rho_{0}=q=(\beta+1) / 2$ and $e_{m}$ $=\rho_{1}=p=(\alpha+1) / 2$ respectively, there are regarded as fixed some $p+1, p \geqq 1$, of the points $\xi_{1}, \xi_{2}, \cdots, \xi_{m}, a_{0}, a_{1}, \cdots, a_{p}$ say, -1 $=a_{0}<a_{1}<\cdots<a_{p-1}<a_{p}=+1$, loaded with the charges $\rho_{0}, \rho_{1}, \cdots$, $\rho_{p}$ respectively, the remaining points $x_{1}, x_{2}, \cdots, x_{n}, n=m-p-1$ being variable in $I$ and loaded all with the charge 1 . We determine now the generalized Jacobi polynomials, $P_{n}^{\left(\alpha_{0}, \ldots, \alpha_{p}\right)}(x), \alpha_{i}=2 \rho_{i}-1$, $i=0, \cdots, p$, called also Heine-Stieltjes polynomials, of the degree $n$ and the order $p$, by the condition that their zeros $x_{1}^{(n)}, x_{2}^{(n)}, \cdots$, $x_{n}^{(n)}$ are those values of the variables $x_{1}, x_{2}, \cdots, x_{n}$ which maximize globally or locally the absolute value of the above Vandermondean on $I$, by the mentioned side-conditions. The distribution of the variable points $x_{1}, x_{2}, \cdots, x_{n}$ in the intervals $\left(a_{0}, a_{1}\right),\left(a_{1}, a_{2}\right), \cdots,\left(a_{p-1}, a_{p}\right)$ characterized by the "type" $\left(n_{1}, n_{2}, \cdots, n_{p}\right)$, where $n_{i}$ denotes the number of the points located in the subinterval $\left(a_{i-1}, a_{i}\right)$ of $I$, is wholly arbitrary, and there are, accordingly, at least $\sigma=C_{m-2, n}$ $=C_{m-2, p-1}$ Heine-Stieltjes polynomials of the degree $n$ and the order $p .{ }^{5}$ It was asserted by Heine $e^{6}$ and proved by Stieltjes ${ }^{7}$ (essentially)

2 We use, by the principle of economy in means of notation, the same letter $P$ in the new denoting of the Jacobi polynomials as in the old one, though $\alpha_{0} \neq \alpha$ and $\alpha_{1} \neq \beta$ (but $\alpha_{0}=\beta$ and $\alpha_{1}=\alpha$ ).

${ }^{3} \mathrm{By}$ the same principle as in 2 we use the same notation $x_{1}, x_{2}, \cdots, x_{n}$ for the zeros of the Jacobi polynomial $P_{n}^{\left(\alpha_{0}, \alpha_{1}\right)}(x)$ as for the zeros of the Jacobi polynomial $P_{n}^{(\alpha, \beta)}(x)$ though the order of the first zeros is reversed in comparison with the second zeros. A confounding is avoidable by some careful attention.

${ }^{4}$ G. Szegö, 1.c., p. 136.

${ }^{5}$ G. Szegö, 1.c., p. 147.

${ }^{6}$ E. Heine, Handbuch der Kugelfunctionen, vol. 1, Berlin 1878, pp. 472-479.

7 T. J. Stieltjes, Sur certaines polynomes qui verifient une équation differentielle linéaire du second ordre et sur la theorie des fonctions de Lame, Acta Math. vol. 6 (1885) pp. 321-326. 
and by Szegö ${ }^{8}$ that there are exactly $\sigma$ Heine-Stieltjes polynomials of the mentioned degree and order, one of each type. Stieltjes and Szegö use for this uniqueness proof the differential equation of the second order of these polynomials

$$
A(x) y^{\prime \prime}+2 B(x) y^{\prime}+C(x) y=0
$$

where $A(x)=\left(x-a_{0}\right)\left(x-a_{1}\right) \cdots\left(x-a_{p}\right)$ and $B(x)=A(x) A_{1}^{\prime}(x) / A_{1}(x)$, with $A_{1}(x)=\left(x-a_{0}\right)^{\rho_{0}} \cdots\left(x-a_{p}\right)^{\rho_{p}}$ and where $C(x)$ is a polynomial of the degree $p-1$, such that the differential equation (1) has a polynomial solution of the degree $n$.

3. The generalized orthogonality relation, which the HeineStieltjes-polynomials satisfy. We derive now the identity announced in the title of this note, valid for any two different Heine-Stieltjes polynomials $y$ and $z$, by eliminating the polynomial $C(x)$ from the differential equation (1). The idea of such an elimination was suggested to the author by Professor S. Agmon from the Hebrew University in Jerusalem, and the proof given here is of the referee of this paper, the original proof of the author being much longer.

Let $(C(x) ; y)$, where $C(x)=s_{0} x^{p-1}+\cdots+s_{p-1}$ and $(D(x) ; z)$ where $D(x)=s_{0}^{\prime} x^{p-1}+\cdots+s_{p-1}^{\prime}$ represent two different "solutions" of the above differential equation (1). ${ }^{9}$ Then $C(x) \neq D(x)$, since, as proved in Szegö, ${ }^{10}$ (1) cannot have two polynomial solutions $y$ and $z$ linearly independent of each other, for a given $C(x)$. We conclude, as in Szegö, ${ }^{11}$ that

$$
\frac{d}{d x}\left\{H(x)\left(y^{\prime} z-y z^{\prime}\right)\right\}=\frac{D(x)-C(x)}{A(x)} y z H(x) .
$$

Here $H(x)=\prod_{\nu=0}^{p}\left|x-a_{\nu}\right|{ }^{2_{\nu}}$. Since $H\left(a_{k}\right)=0, k=0,1, \cdots, p$, we obtain

$$
\int_{a_{k}}^{a_{k+1}} \frac{D(x)-C(x)}{A(x)} y z H(x) d x=0, \quad k=0,1, \cdots, p-1 .
$$

This is a system of homogeneous linear equations for the quantities $s_{i}-s_{i}^{\prime}, i=0, \cdots, p-1$; hence writing

$$
\begin{aligned}
\int_{a_{k}}^{a_{k+1}} y z \cdot x^{l}\left|\left(x-a_{0}\right)^{\alpha_{0}} \cdots\left(x-a_{p}\right)^{\alpha_{p}}\right| d x & =A_{k l} ; \\
k, l & =0,1, \cdots, p-1,
\end{aligned}
$$

\footnotetext{
${ }^{8}$ G. Szegö, 1.c., p. 150.

${ }^{9}$ G. Szegö, 1.c., p. 150.

${ }^{10}$ G. Szegö, l.c., p. 148.

${ }^{11}$ G. Szegö, l.c., p. 150.
} 
the determinant $\left|A_{k l}\right|$ must vanish. This is the announced identity, connecting two different (not linearly dependent) Heine-Stieltjes polynomials $P_{n}^{\left(\alpha_{0}, \cdots, \alpha_{p}\right)}(x)$ and $P_{n^{\prime}}^{\left(\alpha_{0}, \cdots, \alpha_{p}\right)}(x), n \neq n^{\prime}$, or $n=n^{\prime}$, but $\left(n_{1}, n_{2}, \cdots, n_{p}\right) \neq\left(n_{1}^{\prime}, n_{2}^{\prime}, \cdots, n_{p}^{\prime}\right)$. For $p=1$ (2) goes over in the known orthogonality relations

$$
\int_{-1}^{+1} P_{n}^{\left(\alpha_{0}, \alpha_{1}\right)}(x) \cdot P_{n^{\prime}}^{\left(\alpha_{0}, \alpha_{1}\right)}(x)\left|(x+1)^{\alpha_{0}}(x-1)^{\alpha_{1}}\right|=0
$$

with $\left|(x+1)^{\alpha_{0}}(x-1)^{\alpha_{1}}\right|$ as weight function. We call $\mid\left(x-a_{0}\right)^{\alpha_{0}} \ldots$ $\left(x-a_{p}\right)^{\alpha_{p}} \mid$ still the weight function of the generalized Jacobi polynomials of the order $p$.

By introduction of the extended Heine-Stieltjes polynomials $p_{m}^{\left(\alpha_{0}, \cdots, \alpha_{p}\right)}(x)=\left(x-a_{0}\right)^{\alpha_{0}} \cdots\left(x-a_{p}\right)^{\alpha_{p}} P_{n}^{\left(\alpha_{0}, \cdots \alpha_{p}\right)}(x)$ and by denoting $\int_{\alpha_{k}}^{\alpha_{k}+1}\left(u v x^{l} /\left(x-a_{0}\right)^{\alpha_{0}} \cdots\left(x-a_{p}\right)^{\alpha_{p}}\right) d x$, by $B_{k l}$, we obtain $\left|B_{k l}\right|=0 .{ }^{12}$

We turn finally to the special case, interesting among others for the theory of the transfinite diameter and the Fekete-diameters of a finite degree $m$, that $\alpha_{i}=\rho_{i}=1, i=0, \cdots, p$. Then the generalized Vandermondean $V_{m}\left(\xi_{1}, \xi_{2}, \cdots, \xi_{m} ; e_{1}, e_{2}, \cdots, e_{m}\right)$ becomes the usual Vandermondean $V_{m}\left(\xi_{1}, \xi_{2}, \cdots, \xi_{m}\right)=\prod_{1 i_{i<k} m}\left(\xi_{i}-\xi_{k}\right)$ and, since $A_{1}(x)=A(x)$, there results $B(x)=A^{\prime}(x)$.

Mathematical Institute of the Technical University of Berlin

$12 u$ and $v$ are two different extended Heine-Stieltjes polynomials with the same superscripts $\alpha_{0}, \alpha_{1}, \cdots, \alpha_{p}$. The weight-function corresponding to them is $\left|\left(x-a_{0}\right)^{-\alpha_{0}}\left(x-a_{1}\right)^{-\alpha_{1}} \cdots\left(x-a_{p}\right)^{-\alpha_{p}}\right|=\left|\left(x-a_{0}\right)^{\alpha_{0}} \cdots\left(x-a_{p}\right)^{\alpha_{p}}\right|^{-1}$.

\section{AN INTEGRAL TRANSFORMATION RELATION}

\section{R. G. BUSCHMAN}

In a recent paper Rooney [3] has discussed the interesting formula

$$
\int_{0}^{\infty} t^{\nu+1} K_{\nu}(s t) \phi\left(t^{2}\right) d t=2^{\nu-1} s^{-\nu} \int_{0}^{\infty} e^{-y} y^{\nu-1} f\left(s^{2} / 4 y\right) d y
$$

as a generalization of the formula for the Laplace transformation of $t^{\nu} \phi\left(t^{2}\right)$. Here $K_{\nu}(x)$ is the modified Bessel function of the second kind and

$$
f(s)=\int_{0}^{\infty} e^{-s t} \phi(t) d t=\mathscr{L}\{\phi(t)\}
$$

Received by the editors March 12, 1958 and, in revised form, May 7, 1958. 